\title{
Near-field Characterization on Light Emanated from Subwavelength Plasmonic Double Slit of Finite Length
}

\author{
Ki Young Kim ${ }^{1}$, Anatoliy V. Goncharenko ${ }^{1,3}$, Jian-Shiung Hong ${ }^{2,3}$, and Kuan-Ren Chen ${ }^{1,2, *}$ \\ ${ }^{1}$ Department of Physics, National Cheng Kung University, Tainan 701, Taiwan \\ ${ }^{2}$ Institute of Electro-Optical Science and Engineering, National Cheng Kung University, Tainan 701, Taiwan \\ ${ }^{3}$ Advanced Optoelectronic Technology Center, National Cheng Kung University, Tainan 701, Taiwan
}

(Received February 15, 2011 : revised May 4, 2011 : accepted May 27, 2011)

\begin{abstract}
Near-field properties of light emanated from a subwavelength double slit of finite length in a thin metal film, which is essential for understanding fundamental physical mechanisms for near-field optical beam manipulations and various potential nanophotonic device applications, is investigated by using a threedimensional finite-difference time-domain method. Near-field intensity distribution along the propagation direction of light after passing through the slit has been obtained from the phase relation of transverse electric and magnetic fields and the wave impedance. It is found that the near field of emerged light from the both slits is evanescent, that is consistent with conventional surface plasmon localization near the metal surface. Due to the finite of the slit, the amplitude of this evanescent field does not monotonically approach to than of the infinite slit as the slit length increases, i.e. the near-field of the longer slit along the center line can be weaker than that of the shorter one.
\end{abstract}

Keywords : Subwavelength slit, Plasmonics, Near-field characterization, Finite-difference time-domain (FDTD) method

OCIS codes : (250.5403) Plasmonics; (310.6628) Subwavelength structures, nanostructures

\section{INTRODUCTION}

The famous experiment on the interference of light diffracted by a double slit was performed by Thomas Young, who found that unique interference patterns were related to light sources at a specific separation distance between two slits $[1,2]$. This experiment also inspired the development of the particle-wave duality [3], which became again a trigger for building up the concepts in quantum mechanics. Although this experiment was initially performed more than two centuries ago, even nowadays, intensive research activity on double slit interference is still in progress to explore some novel physical phenomena, including for instance, the light interference characteristics due to a coherence degree of two light sources [2, 4-8], analyses of geometrical effects leading to enhanced light transmission and propagation [9-11]. Recent significant attention to the surface plasmons [12] has been also related to the double slit interference. The interference pattern produced by the double slit in a plasmonic metal film is quite different from the traditional ones due to the surface plasmon generation and localization near the metal surface at optical wavelengths [2, 4-7, 13-15]. While the plasmonic double slits have also been utilized for novel subwavelength optics applications such as unidirectional surface plasmon excitation [16, 17], optical beam manipulations [18, 19], and sub-diffraction limited optical spot generation in the intermediate field region $[20,21]$, the studies on the a subwavelength double slit are still inactive compared with those on the single slit structures. Furthermore, most work done on subwavelength slit structures has been limited to two-dimensional approximations or to very long slits compared with operating wavelengths, though finite slit effect is critical to the light interference in practical structures which is essential in the performance of the subwavelength slit based optical devices.

In this work, fundamental near-field behavior of light diffracted by a subwavelength double slit of finite length in a a thin metal film investigated using an intensive threedimensional finite-difference time-domain (3D FDTD) method [22]; in particular, the near-field intensity distributions are

\footnotetext{
*Corresponding author: chenkr@mail.ncku.edu.tw

Color versions of one or more of the figures in this paper are available online.
} 
obtained from phase relation and field peak ratio between the the electric and magnetic fields transverse to propagation direction. Analysis of the finite slit length effect on the near-field intensity distributions and the comparison of those with two-dimensional infinite slit length case [23] allow us to gain further insights into the physics of the subwavelength plasmonic double slit, which can be potentially useful for various optical devices dealing with subwavelength optical beam manipulation or light focusing in the near-field region.

\section{SUBWAVELENGTH PLASMONIC DOUBLE SLIT OF FINITE LENGTH AND 3D FDTD SIMULATION SETUP}

Figure 1 shows a schematic of the subwavelength double slit with finite slit length, denoted as $s l$, perforated in a thin metal film placed in air. Without loss of generality, the $x$-polarized monochromatic plane wave of $\lambda=633 \mathrm{~nm}$ wavelength is normally incident upon a thin metal film in the $-y$ direction from the upper region. Individual slit width is $80 \mathrm{~nm}$ which is much smaller than the incident wavelength. The metal used in this numerical experiment is silver $(\mathrm{Ag})$ with the dielectric constant of $\mathcal{E}_{r}=-15.91+$ $i 1.07$ at $633 \mathrm{~nm}$ [24]. Due to this negative value of the real part of the dielectric constant, surface plasmons can

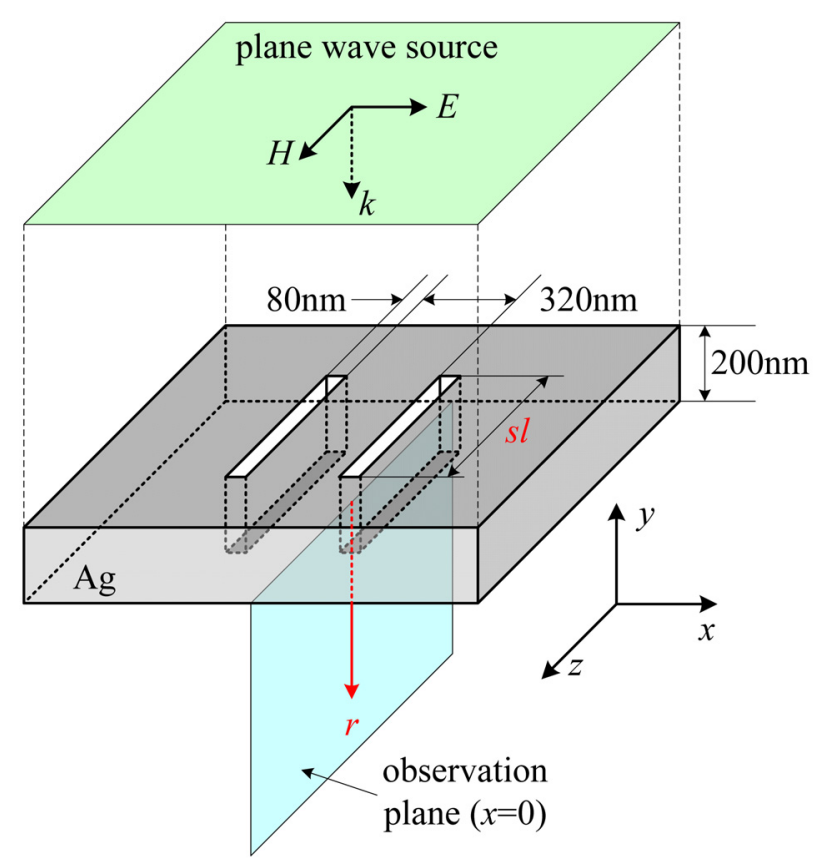

FIG. 1. Schematic of the subwavelength double slit of finite length in thin metal film. The observation plane (the $y z$-plane at $x=0$ ) in which electromagnetic field distributions are going to be obtained is shown below the metal film. The propagation distance $r$ originates from the bottom surface of the metal film. be supported at the interface between the bottom surface of the silver film and air, which is one of the reasons that the near-field characteristics are different from those of conventional double slit in a perfect electric conductor (PEC). Thickness of the metal film is $200 \mathrm{~nm}$, which is thick enough to block the light passing directly through the metal film and at the same time it is also less than half-the-wavelength to eliminate the Fabry-Perot resonance in the longitudinal $y$-direction inside the slits [25]. The slit separation is $320 \mathrm{~nm}$, which is about half-the-wavelength, where optical transmission is known to be stronger than those for other separation distances between the slits [26, 27]. After passing through each slit, the light emerges at the center position between slits, i.e. at the observation plane in Fig. 1, due to the interference of light emanated from each slit, which is a merit of using double slit over the single slit in terms of light focusing or manipulating the light on subwavelength scale. The interference pattern formed by this subwavelength double slit can be influenced by the finite length of the slit. So, as one can expect, the near-

field distribution can be changed with respect to the slit length, which is the main focus of this work.

In order to investigate the near-field characteristics of interfered light after passing through the slits in the metal film, the 3D FDTD simulations were performed using a freely available software package [28], in which material dispersion for real metal has been implemented. Entire computation domain has $1400 \times 1400 \times 400$ cells in $x$-, $z$-, and $y$-directions, respectively, with the Yee's spatial unit cell of $5 \mathrm{~nm}$. The perfectly matched layers have been set up at all boundaries for the full-wave simulations.

\section{NUMERICAL RESULTS AND DISCUSSION}

Figure 2 shows snapshots of the $x$-component of the electric field $E_{x}$ and $z$-component of the magnetic field $H_{z}$ for three different slit length cases, in the observation plane shown in Fig. 1. Those are taken when the $H_{z}$ field is close enough to zero at the bottom surface of the silver film, as indicated in Fig. 2 (a), (b), and (c). Here, both the electric and magnetic fields of the incident plane wave have been normalized to unity. The positions of the slits with different lengths have been denoted as solid lines in the upper region. The numbers on the ordinate are the distances originated from the bottom surface of the silver film, i.e. the distance $r$ shown in the observation plane in Fig. 1. Even when the magnetic field is zero at the bottom surface of the silver film, the electric field is rather far from zero (this will be briefly discussed below), causing the non-zero value of the near-field intensity. Comparing the cases of $s l=600 \mathrm{~nm}$ and $s l=1200 \mathrm{~nm}$ in Fig. 2 (d) and Fig. 2 (e), the intensity of the first focused spot near $r=300 \mathrm{~nm}$ and the field near the bottom surface of the silver film are seen to be stronger for the longer slit case. 

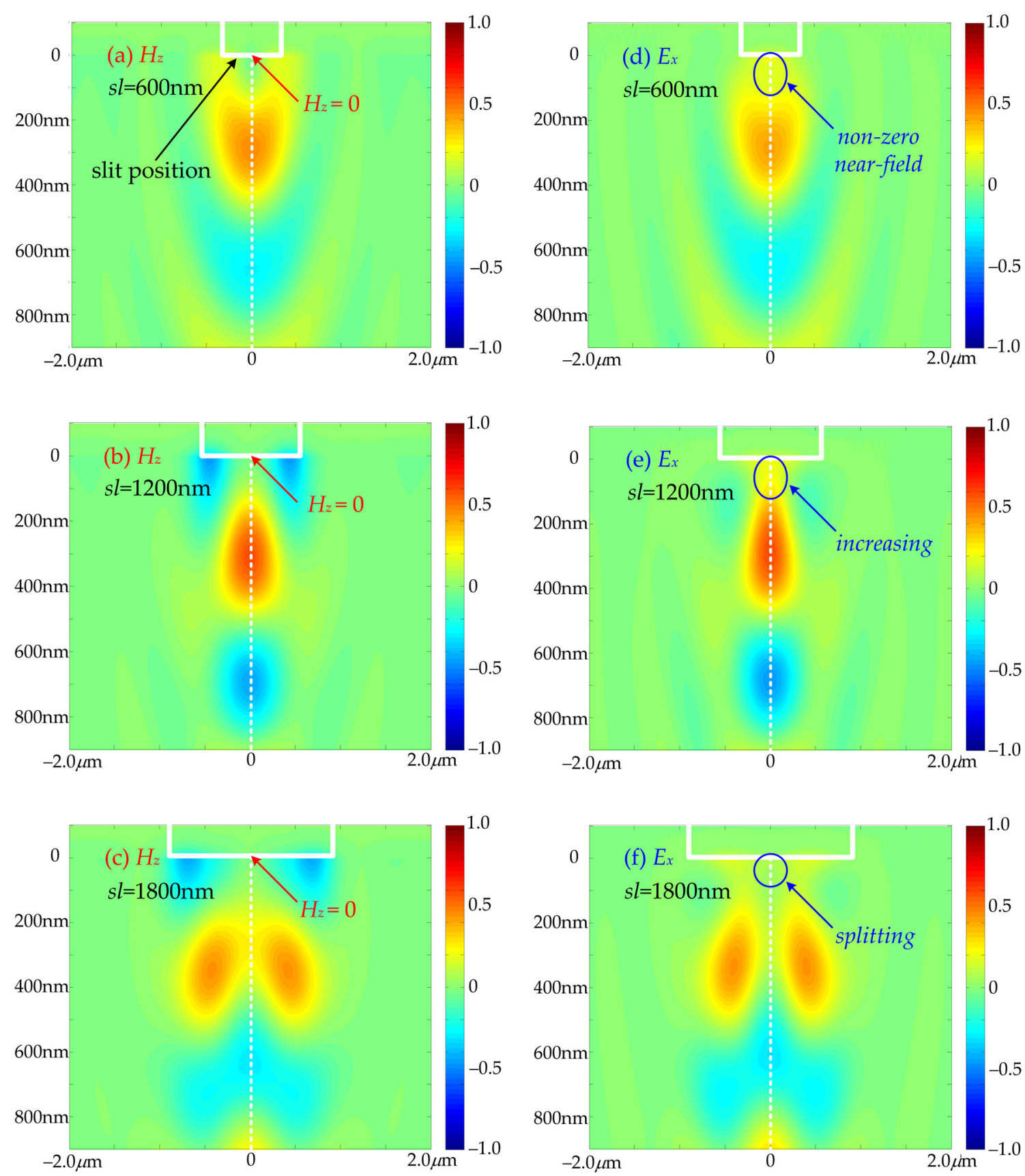

FIG. 2. Snapshots of the electric and magnetic fields when the magnetic fields at the exit of the metal film are close to zero for different slit lengths of $s l=600,1200$, and $1800 \mathrm{~nm}$ in the observation plane as shown in Fig. 1, i.e., horizontal and vertical axes are $z$ - and $y$-positions, respectively. [(a)(b)(c): the $z$-component of the magnetic field $\left(H_{z}\right),(\mathrm{d})(\mathrm{e})(\mathrm{f})$ : the $x$-component of the electric field $\left(E_{x}\right)$, and (a)(d): $s l=600 \mathrm{~nm},(\mathrm{~b})(\mathrm{e}): s l=1200 \mathrm{~nm},(\mathrm{c})(\mathrm{f}): s l=1800 \mathrm{~nm}]$.

However, the focused spot for the $s l=1800 \mathrm{~nm}$ case splits as shown in Fig. 2 (f), and the field intensity along the central line becomes smaller than that in previous (shorter length) cases. This peak splitting is thought to be a result of nonuniform field distribution along the slit direction which in turn can result from the excitation of higher-order $\mathrm{TE}_{\mathrm{m} 0}$ eigenmodes of the cavity. In other words, the splitting can be considered as resulting from the interaction of fields emanated from each slit, while these fields are associated with corresponding eigenmodes and hence should depend on the slit length. More detailed discussion on this splitting for the finite length slit and its physical mechanism will be reported elsewhere. Obviously, a correct treatment of the problem should include such phenomena as near-field interaction between modes and mode mixing.

Figure 3 shows the intensities of the $E_{x}$ and $Z H_{z}$ fields versus the normalized propagation distance $k r$, where $Z$ is the complex wave impedance and $k$ is the free space wave 

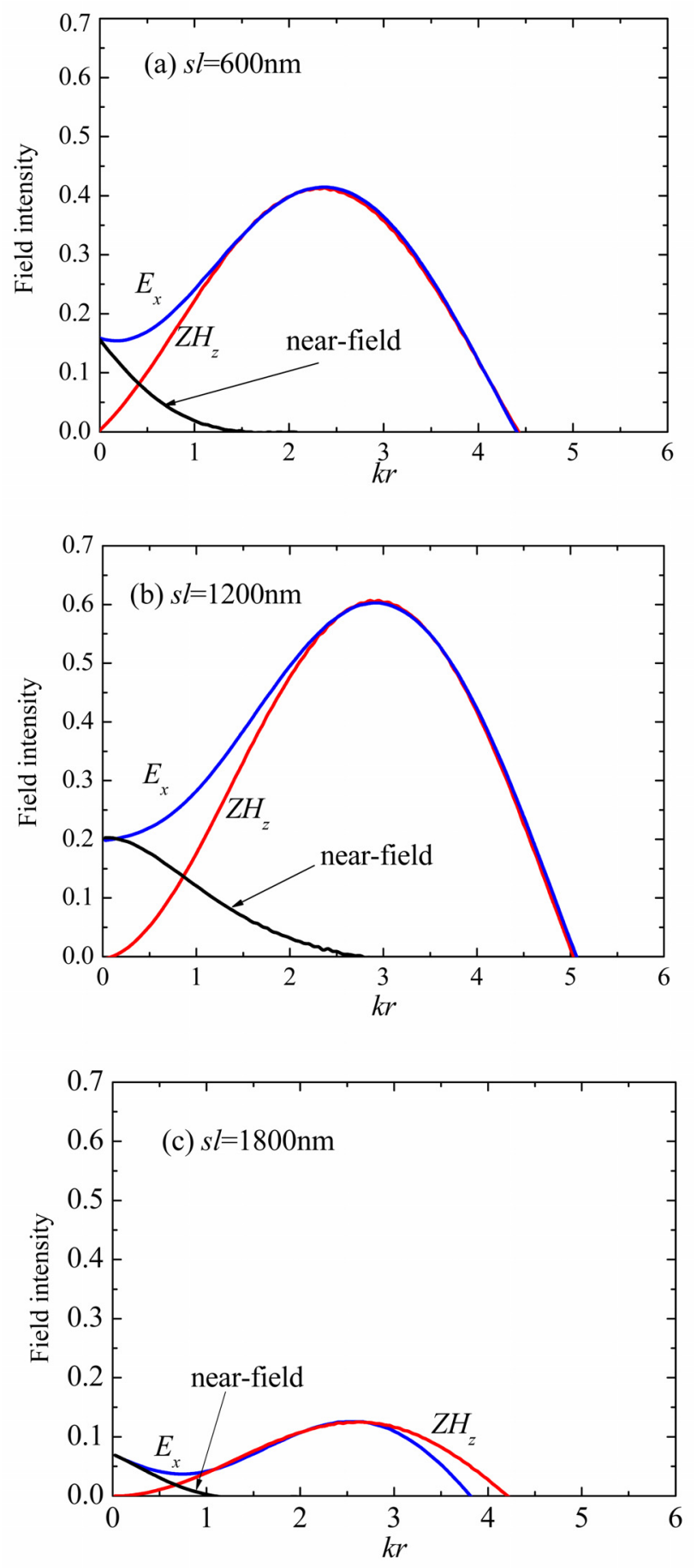

FIG. 3. The $E_{x}, H_{z}$, and near-field intensities along the propagation direction at the center position $(z=0)$ in the observation plane (shown in Fig. 1) for (a) $s l=600 \mathrm{~nm}$, (b) $s l=1200 \mathrm{~nm}$, and (c) $s l=1800 \mathrm{~nm}$. The near-field intensity distributions along the propagation direction extracted from the subtraction between the $E_{x}$ and $Z H_{z}$ fields.

number, at the center position $(z=0)$ in the observation plane shown in Fig. 1 and also denoted as dotted lines in Fig. 2. In the near-field region, the electric and magnetic fields are out of phase. However, beyond the near-field region, the ratio and the locations of their peaks allow us to determine the impedance $Z$, since the focused $E_{x}$ field has similar distributions with the $H_{z}$ field and they are expected to be almost in phase [29]. Because the instants when the $H_{z}$ fields are close to zero at the bottom surface of the silver film have been taken as shown in Fig. 2, the $Z H_{z}$ field can be used to estimate the $E_{x}$ field distributions in the near zone without the near-field contributions (the real $E_{x}$ field includes inherent near-field contributions from the surface plasmon supported by the silver / air interface). Thereby, the evanescent intensity distributions along the normalized propagation distance $\mathrm{kr}$ can be extracted from the difference between the $E_{x}$ and $Z H_{z}$ fields as shown in Fig. 3. According to this procedure, the non-zero evanescent fields have been obtained. That is a major difference from the case of the PEC film, i.e. the evanescent field intensity in the case of the PEC film is nearly negligible [23]. While the $E_{x}$ and $Z H_{z}$ field profiles in Fig. 3 (a) and Fig. 3 (b) above the peak positions are almost the same as expected, a deviation between both fields for the $s l=1800$ $\mathrm{nm}$ case is seen about $k r>3.0$ as shown in Fig. 3 (c). This is probably because the peaks are split and the focusing at the center position is not very strong as compared with the previous two shorter slit cases as shown in Fig. 2 (c) and 2 (f).

For the two-dimensional case, i.e. infinite slit length, the electromagnetic field distributions are supposed to be uniform at the same lateral position because there is no dependence along the $z$-direction. However, in the current three-dimensional case of finite length slit, the electromagnetic field distributions are seen to be changed due to the finite slit length even though measured at the same position, e.g., here at the central line in the observation plane. It is probably because the surface charge and surface current distributions at the bottom surface of the silver film are not uniform any more along the slit length direction due to the finite length of the slit, which eventually makes the light interference more complex than that in the two-dimensional infinite slit length case. The obtained near-field intensities decrease evanescently as the normalized propagation distance $k r$ increases and become negligible above certain normalized propagation distance $k r$, that is consistent with conventional near-field localization of the surface plasmons. For $s l=600 \mathrm{~nm}$ (about $1 \lambda$ ), the near-field intensity become negligible at about $k r>1.5$. Meanwhile, for $s l=1200 \mathrm{~nm}$ (about $2 \lambda$ ), the range of the negligible near-field intensity shifts to about $k r>2.8$, meaning stronger surface plasmons are being supported for this slit length. However, for $s l=1800 \mathrm{~nm}$ (about $3 \lambda$ ), the near-field intensity becomes considerably reduced to be even weaker than that for $s l=600 \mathrm{~nm}$ case and the boundary of the negligible near-field intensity range is at $k r>1.1$, which means that the near-field characteristics of the longer but finite slit may not be closer to those of the infinite slit length case. This is because the peaks of the fields at $z=0$ in the observation plane split and shift towards both 


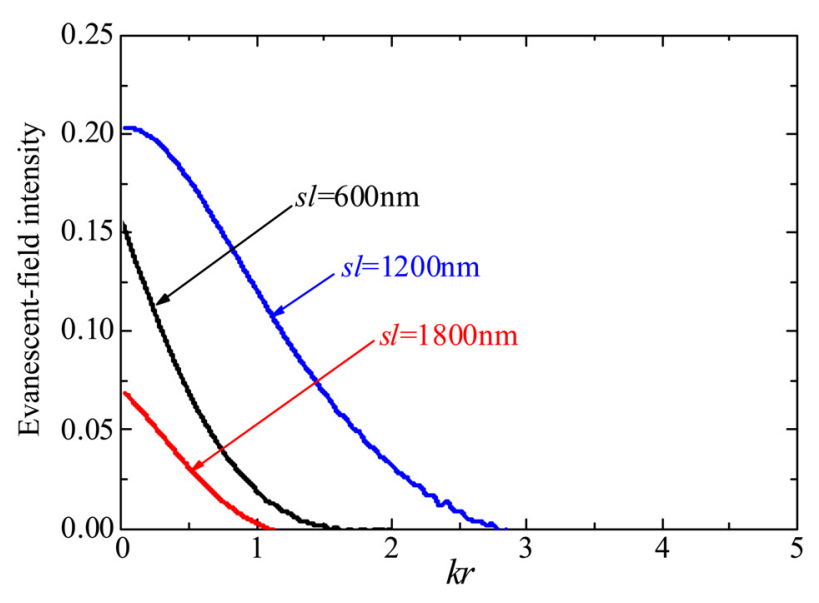

FIG. 4. The evanescent-field intensity obtained as in Fig. 3 along the propagation direction at the center position in the observation plane.

directions along the z-axis due to an oscillation of the fields along $z$-direction as shown in Fig. 2 (c) and Fig. 2 (f), resulting in even weaker focused fields near the center position than those in the previous two cases as mentioned.

Figure 4 shows the evanescent field intensities, though each of them is already shown separately in Fig. 3, for a clearer comparison with each case. The evanescent field intensities at the bottom surface of the silver film, i.e., at $r=0$, vary with the slit length and are also stretching with the distance $r$ to the propagation direction but decrease evanescently when $k r$ increases as mentioned above. The evanescent field intensity for $s l=1200 \mathrm{~nm}$ (about $2 \lambda$ ) is stronger than for the other two cases and is stretched further also as compared with the $s l=600 \mathrm{~nm}$ and $1800 \mathrm{~nm}$ cases; it is also closer to that of the two-dimensional infinite slit length case [23] as compared with the other two cases.

Such a behavior of the evanescent field intensity can be a result of the contribution of higher-order eigenmodes. In fact, as the slit length increases, more and more higher order modes can occur in the cavity. Obviously, the odd modes are favorable for increasing the evanescent field at the center, while the even modes are favorable to decrease it. As the slit length approaches infinity, the evanescent field intensity should naturally approach to that of two-dimensional case. However, this approaching is oscillating rather than monotonic because it involves more and more higher order eigenmodes.

\section{CONCLUSION}

In conclusion, the near-field characteristics of light from a subwavelength double slit of finite length in a thin metal film have been investigated by using 3D FDTD method. Near-field intensity along the symmetry axis at the center position of the slit direction has been obtained with phase relation of the transverse electric and magnetic fields and the ratio between them. It is found that the evanescent field distribution is strongly dependent on the slit length. As is shown, the evanescent field intensity distribution along the propagation direction may not necessarily increase toward that of the ideal two-dimensional infinite slit length case as the slit length increases. That might be significant in terms of designing practical subwavelength metal slit based plasmonic devices and the fundamental physics behind it as well. This effect may be attributed to the contribution of higher-order eigenmodes of the cavity, which are capable of critically changing the field distribution in the near zone.

\section{ACKNOWLEDGMENT}

This work was supported by the Ministry of Education, Taiwan, R.O.C., under the NCKU Project of Promoting Academic Excellence \& Developing World Class Research Centers and the National Science Council. We are grateful to the National Center for High-Performance Computing for the computer time and facilities.

\section{REFERENCES}

1. T. Young, "Experiments and calculations relative to physical optics," Phil. Trans. R. Soc. Lond. 94, 1-16 (1804).

2. T. D. Visser, "Young's interference experiment: the long and short of it," in Tribute to Emil Wolf: Science and Engineering Legacy of Physical Optics, T. P. Jannson ed. (SPIE, Bellingham, USA, 2005), Chapter 15.

3. R. P. Feymann, R. L. Leighton, and M. Sanders, The Feymann Lectures on Physics (Addison-Wesley, MA, USA, 1965).

4. H. F. Schouten, N. Kuzmin, G. Dubois, T. D. Visser, G. Gbur, P. F. A. Alkemade, H. Blok, G. W.’t Hooft, D. Lenstra, and E. R. Eliel, "Plasmon-assisted two-slit transmission: Young's experiment revisited," Phys. Rev. Lett. 94, 053901 (2005).

5. C. H. Gan, G. Gbur, and T. D. Visser, "Surface plasmons modulate the spatial coherence of light in Young's interference experiment," Phys. Rev. Lett. 98, 043908 (2007).

6. Z. Li, J.-S. Zhang, H.-F. Yan, and Q.-H. Gong, "Complex modulation in plasmon-assited transmission spectra of a two-slit structure," Chin. Phys. Lett. 24, 3233-3236 (2007).

7. N. Kuzmin, G. W.'t Hooft, E. R. Eliel, G. Gbur, H. F. Schouten, and T. D. Visser, "Enhancement of spatial coherence by surface plasmons," Opt. Lett. 32, 445-447 (2007).

8. T. D. Visser and R. W. Schoonover, "A cascade of singular field patterns in Young's interference experiment," Opt. Comm. 281, 1-6 (2008).

9. R. Welti, "Light transmission through two slits: the Young experiment revisited," J. Opt. A: Pure Appl. Opt. 8, 606-609 (2006).

10. R. Gordon, "Near-field interference in a subwavelength double slit in a perfect conductor," J. Opt. A: Pure Appl. Opt. 8, L1-L3 (2006). 
11. K.-M. Chae, H.-H. Lee, S.-Y. Yim, and S.-H. Park, "Evolution of electromagnetic interference through nano-metallic double-slit," Opt. Express 12, 2870-2879 (2004).

12. H. Raether, Surface Plasmons on Smooth and Rough Surfaces and on Gratings (Springer, New York, USA, 1988).

13. S. Ravets, J. C. Rodier, B. E. Kim, J. P. Hugonin, L. Jacubowiez, and P. Lalanne, "Surface plasmons in the Young slit-doublet experiment,” J. Opt. Soc. Am. B 26, B28-B33 (2009).

14. P. Ginzburg, E. Hirshberg, and M. Orenstein, "Rigorous analysis of vectorial plasmonic diffraction: single- and double-slit experiments," J. Opt. A: Pure Appl. Opt. 11, 114024 (2009).

15. R. Zia and M. L. Brongersma, "Surface plasmon polariton analogue to Young's double-slit experiment," Nat. Nanotechnol. 2, 426-429 (2007).

16. T. Xu, Y. Zhao, D. Gan, C. Wang, C. Du, and X. Luo, "Directional excitation of surface plasmons with subwavelength slits," Appl. Phys. Lett. 92, 101501 (2008).

17. Y. Wang, L. L. Wang, J. Q. Liu, X. Zhai, L. Wang, D. Xiang, Q. Wan, and B. Meng, "Plasmonic surface-wave bidirectional splitter in different angles of incident light," Opt. Comm. 283, 1777-1779 (2010).

18. M. A. Vincenti, A. D’Orazio, M. Buncick, N. Akozbek, M. I. Bloemer, and M. Scalora, "Beam steering from resonant subwavelength slits filled with a nonlinear material," J. Opt. Soc. Am. B 26, 301-307 (2009).

19. G.-G. Zheng and X.-Y. Li, "Optical beam manipulation through two metal subwavelength slits surrounded by dielectric surface gratings,” J. Opt. A: Pure Appl. Opt. 11, 075002 (2009).

20. K. R. Chen, "Focusing of light beyond the diffraction limit of half the wavelength," Opt. Lett. 35, 3763-3765 (2010). 21. K. R. Chen, W. H. Shu, H. C. Fang, C. P. Liu, C. H. Huang, H. C. Chui, C. H. Chuang, Y. L. Lo, C. Y. Lin, S. J. Chang, F. Y. Hung, H. H. Hwuang, and A. Y.-G. Huh, "Beyond-limit light focusing in the intermediate zone," arXiv:0901.1731.

22. K. S. Yee, "Numerical solution of initial boundary value problems involving Maxwell's equations in isotropic media," IEEE Trans. Antennas Propag. 14, 302-307 (1966).

23. K. R. Chen, K. Y. Kim, J. S. Hong, A. V. Goncharenko, and $\mathrm{K}$. J. Lee, "Near-field characterization on light emanated from subwavelength plasmonic double slits," in Proc. 4th Int. Conf. Electromagnetic Near Field Characterization and Imaging (Taipei, Taiwan, 2009), pp. 219-223.

24. E. D. Palik, Handbook of Optical Constants of Solids (Academic Press, New York, USA, 1998).

25. J. Wuenschell and H. K. Kim, "Excitation and propagation of surface plasmons in metallic nanoslit structure," IEEE Trans. Nanotech. 7, 229-236 (2008).

26. M. Mansuripur, Y. Xie, A. R. Zakharian, and J. V. Moloney, "Transmission of light through slit apertures in metallic films," IEEE Trans. Magnetics 41, 1012-1015 (2005).

27. Y. Xie, A. R. Zakharian, J. V. Moloney, and M. Mansuripur, "Transmission of light through slit apertures in metallic films," Opt. Express 12, 6106-6121 (2004).

28. A. F. Pskooi, D. Roundry, M. Ibanescu, P. Bermel, J. D. Joannopoulos, and S. G. Johnson, "MEEP: a flexible freesoftware package for electromagnetic simulations by the FDTD method," Comp. Phys. Commun. 181, 687-702 (2010).

29. J. D. Jackson, Classical Electrodynamics, 3rd ed. (John Wiley \& Sons Inc., New York, USA, 1998). 